\title{
Evasion of COPD in smokers: at what price?
}

\author{
Manuel G. Cosio*,\# and Marina Saetta ${ }^{\#}$
}

\begin{abstract}
Investigations toward the understanding of chronic obstructive pulmonary disease (COPD) have been directed, so far, to the study of mechanisms leading to the disease. We believe that understanding why $\sim 80 \%$ of smokers evade COPD and how this evasion is accomplished might be a fruitful endeavour that could advance knowledge of the development of the disease. Since the inflammatory infiltrate smokers develop seems to be the key element leading to the lung destruction in COPD, the understanding of the possible ways inflammation can be dampened, as well as its consequences, ought to be important. We review here some of the mechanisms by which inflammation is controlled: by the post-translational regulons, by the mechanisms preventing full activation of dendritic cells and by the regulatory T-cells. The potential role of the M2 alveolar macrophage phenotype and the newly described myeloid-derived suppressor cells is mentioned. We also point out that evasion comes at a price, as healthy smokers might be immunosuppressed to some extent and unable to prevent the development of cancer, certainly less so than in severe COPD, where immunity is heightened. Probably, the knowledge of the mechanisms of evasion from COPD could add significantly to the understanding of those leading to the disease.
\end{abstract}

KEYWORDS: Cancer, chronic obstructive pulmonary disease, immune response, monocyte/ macrophage

$\mathrm{t}$ is clear that smokers are at risk of developing chronic obstructive pulmonary disease (COPD) and, in some cases, severe COPD. Many theories have been proposed to explain the mechanisms and development of the disease; however, little attention is being paid to why and how the majority of smokers do not develop or "evade" COPD, or only develop a mild disease. Perhaps we should be changing tactics in our approach to the study of the mechanisms of COPD development and start paying more attention to the other side of the coin: what are the mechanisms that allow the majority of smokers to evade COPD and is there a price to pay for this evasion?

In this Perspective we examine the mechanisms involved in the essential process of limiting or dampening inflammation, a cardinal feature in the pathogenesis of COPD. These mechanisms are essential in order to evade tissue damage and to allow the initiation of repair processes. Possibly, if we could understand the mechanisms of evasion, we could more easily understand why some smokers progress toward the development of COPD.
We have recently proposed that uncontrolled adaptive immune inflammation, triggered by the innate inflammation initiated by smoking, evolves into an autoimmune reaction that destroys the lung, causing COPD, particularly severe COPD, in some smokers [1]. Because the adaptive immune response and autoimmunity could help us to understand not only the mechanisms for the development of the disease but also the mechanisms for the evasion of it, it will be useful to look first at the build-up of the adaptive immune response and autoimmunity responsible for the development of COPD.

The initial innate inflammation elicited by epithelial injury and chronically maintained by constant smoke exposure is the key factor for the eventual development of the disease, unless this inflammatory reaction is controlled and/or minimised. The inflammation is important because it injures the lung tissue, releasing or producing self antigens with the potential of triggering an adaptive immune reaction involving $\mathrm{CD} 8+$ and $\mathrm{CD} 4+\mathrm{T}$ cells and B-cells that, if not properly controlled, would, over time, induce the pathological lung abnormalities that are the basis of COPD [1].

\section{AFFILIATIONS}

${ }^{*}$ Respiratory Division and MeakinsChristie Laboratories, Royal Victoria Hospital, Dept of Medicine, McGill University, Montreal, QC, Canada. \#Dept of Cardiac, Thoracic and Vascular Sciences, Section of Respiratory Diseases, University of Padua, Padua, Italy.

CORRESPONDENCE M.G. Cosio Clinica Pneumologica Universita' di Padova Via Giustiniani 3 Padova 35128 Italy E-mail: manuel.cosio@mcgill.ca

Received:

Aug 082011

Accepted after revision:

Oct 042011

First published online:

Oct 172011 


\section{WHY DO SO MANY SMOKERS EVADE COPD?}

If inflammation is key in the development of COPD and if an adaptive immune response directed against lung self antigens drives the persistent inflammatory response found in smokers, we have a starting point for our discussion and, possibly, for the understanding of the evasion phenomenon. The development of an adaptive immune response to a self antigen, along with the eventual development of autoimmunity, would depend on the level of tolerance to the antigen: when tolerance is high, no adaptive inflammation would result; when there is no tolerance, a full adaptive immune reaction and autoimmunity would develop. However, tolerance to antigens, especially self antigens, is not an all-or-nothing phenomenon [2]. Different degrees of tolerance can exist, giving different degrees of inflammatory response to an antigenic challenge and, hence, different degrees of disease severity. Different degrees of tolerance failure to self or modified self antigens could explain the variable responses of the lung to cigarette exposure, the variable number of T-cells in the lung of smokers, the wide range of forced expiratory volume in $1 \mathrm{~s}$ (FEV1) found in smokers and, thus, the correlation found between the numbers of T-cells and the extent of the disease reported in smokers $[3,4]$.

\section{HOW IS TOLERANCE LEVEL DETERMINED?}

Central thymic tolerance, through the elimination of selfreactive T-cells and the production of "natural" CD4+ regulatory T-cells (Tregs), is the first and essential mechanism for immune regulation and immune tolerance [5]. However, the back-up of the peripheral tolerance system is essential. There is now agreement that professional antigen-presenting cells, of which the dendritic cell (DC) is the main type, are involved in the initiation of both immunity and peripheral tolerance induction [6]. DCs reside in tissues in direct contact with the environment and their role is to collect potential antigens to be presented to T-cells in order to mount an adaptive immune inflammation against those antigens. The ability of DCs to activate T-cells upon antigen presentation depends mainly on the level of inflammation in the microenvironment were the DC resides, the lung in our case. When there is little or no inflammation, an immature or semimature DC might migrate to local lymph tissue, where presentation of antigens from tissue turnover would take place. However, due to the absence of inflammatory stimuli, the DCs would not express the costimulatory molecules and cytokines necessary for $\mathrm{T}$-cell stimulation. In this situation, self antigen recognition by Tcells presented by immature DCs will induce T-cell anergy or/ and Tregs. Anergic T-cells will be unable to proliferate and produce interleukin (IL)-2 in a subsequent challenge by a competent DC and the presence of Tregs would control the effectiveness of possible activated T-cells, thus contributing to the maintenance of tolerance $[6,7]$.

In contrast, when the lung is inflamed, the necessary factors for the recruitment, activation and maturation of the local DCs would be provided along with the costimulatory molecules and cytokines necessary for the activation of T-cells upon antigen presentation. In this situation T-cells would undergo clonal expansion and migrate either to B-cell areas, to assist in antibody production, or to tissues with active inflammation. For these reasons a coordinated migration and maturation of DCs is considered a critical process leading to either tolerance, when the lack of inflammation in the milieu will prevent DC maturation, or immunity, when the strong inflammatory milieu will favour DC maturation, which makes the DC responsible for the final outcome of the immune response [6,7].

From these arguments it becomes evident that the control of the initial innate inflammatory response, induced in the lung by smoking, could be an essential step in the avoidance or establishment of an adaptive immune response that could lead into COPD. There is ample evidence that healthy smokers have milder lung inflammation $[3,4]$ and mice exposed to smoke that do not develop emphysema have no lung inflammation [8]. Possibly, the innate inflammation induced by smoking is being actively suppressed, leading to tolerance and evasion of COPD.

\section{HOW COULD THE INFLAMMATION BE SUPPRESSED?}

Although inflammation is an essential defensive and repair component of the innate immune response in multicellular organisms, it can become a double-edge sword, as excessive inflammation can exacerbate tissue damage and produce potential antigens [7]. Although many mechanisms for the regulation of different inflammatory factors have been described, such as IL-1 receptor antagonist to regulate the effects of IL-1 $\beta$, and the negative regulation of the Toll-like and NOD (nuclear oligomerisation domain)-like receptors $[9,10]$, it is possible that all these mechanisms are controlled by newly described mechanisms that can regulate most chemokines, cytokines, proteases and other factors involved in the initiation and maintenance of the inflammation [11]. To limit the undesirable effects of excessive inflammation many stimuli that trigger the transcription and protein translation, which leads to an inflammatory response, simultaneously trigger the transcription of mRNA encoding proteins that, through a complex programme of intracellular signalling, actively resolves inflammation. Furthermore, because long after transcription has ceased transcribed mRNAs can continue to synthesise pro-inflammatory proteins, a system would ideally be needed to modulate the production of these proteins when they are not needed. Such systems have been described and consist in a variety of post-transcriptional control mechanisms, the post-transcriptional regulons, which modulate the expression of many proteins involved in the immune mediated inflammation, in most cases by promoting mRNA decay and/or inhibiting protein translation [11]. In this way, post-transcriptional dampening of protein expression can actively promote the resolution of inflammation to prevent unintended tissue damage.

It has been found that multiple chemokines and their receptors are downregulated by means of inhibition of protein translation, while cytokines and other inflammatory factors, such as IL-1 $\beta$, tumour necrosis factor, interferon- $\gamma$, IL-2 and IL-6, among many others, are controlled by accelerating mRNA decay [11]. Also indirectly controlled by post-transcriptional regulatory mechanisms are the lipid mediators that have essential roles in the initiation (such as prostaglandins and leukotrienes) and resolution (such as lipoxins, protectins and resolvins) of inflammation [11].

In summary, the natural resolution of inflammation seems to be mainly driven by the following three different types of processes [12]. 
1) Removal of the initial stimulus, which is critical. Many chronic ongoing inflammatory conditions are probably driven mostly by the persistence of the offending agent and/or autoimmunity. We have a great example of this problem with the continuous use of cigarettes!

2) Decrease of the pro-inflammatory mediators involved in attracting new inflammatory cells to the lesions and the persistence of the inflammation, i.e. post-transcriptional regulons by promoting mRNA decay and/or inhibiting protein translation.

3) Removal of the inflammatory cells and cell debris to allow the final repair to occur, for example, alveolar macrophages stimulated by hypoxins, resolvins and protectins will clear polymorphonuclear neutrophils (PMNs) and structural cell apoptotic bodies, which induces a potent anti-inflammatory response and promotes the activation of the anti-inflammatory function of transforming growth factor- $\beta$. Defects in apoptotic cell recognition and response by alveolar macrophages could make apoptotic debris antigenic, promoting chronic inflammation and even autoimmunity, as has been shown in systemic lupus erythematosus $[12,13]$. Some or all of these mechanisms ought to be working in many smokers, probably the $80 \%$ that evade COPD; since the inflammatory response in the lungs of these smokers seems to be controlled, the degree of their inflammation is very small when compared with smokers with severe COPD $[3,4]$.

It is still possible that when the inflammatory stimulus continues, as in smokers (see point 1), the mechanisms available cannot control the innate inflammatory response, or succeed in only partial control, resulting in a chronic inflammation that will vary in severity. As a consequence, constant but variable degrees of tissue destruction, production of antigens and degrees of stimulation of DCs with variable degrees of adaptive immune inflammation and severity of COPD will follow. Chronic inflammation would have three possible undesirable effects in smokers: progressive tissue destruction, which would trigger repair and fibrosis; the development of lung cancer; and the possible development of autoimmunity, all of which are certainly a reality for some smokers. However, mechanisms devised to suppress, or at least attempt the suppression of, chronic inflammation, especially the adaptive immune inflammation, are also available and possibly modulate the progression of the disease. The best known mechanism to control the progression of the adaptive immune inflammation is mediated by the CD4+ CD25+ FoxP3+ Tregs [14, 15]. A deficiency of Tregs can impair the immune system tolerance for autoantigens and lead to autoimmune disease [15]. The behaviour of Tregs in COPD is of interest and seems to vary with the site in the lung and severity of the population studied [16]. Long-term smoking exposure, but not the development of airflow obstruction, increases Treg numbers in the lung [17]. As such, when compared with the lungs of nonsmokers, the lungs of smokers with normal lung function or mild functional abnormalities (FEV1 $70 \%$ predicted) have a greater number of Tregs in the bronchoalveolar lavage fluid [17] and in lung lymphoid follicles, but not in the lung parenchyma [18]. In other studies, the lungs of smokers with COPD and emphysema have been found to have fewer Tregs and less FoxP3 mRNA than the lungs of healthy smokers, who had more Tregs than the nonsmokers [19, 20]. Furthermore, smokers with COPD were found to have an upregulation of FoxP3+ T-cells in the large airways, but had a downregulation of FoxP3+ T-cells in the small airways, the main site of pathological involvement in COPD, which correlated with airflow limitation [21]. This suggests that in smokers with normal lung function or mild COPD, Tregs, by controlling the immune reaction, might prevent the development of severe disease. A failure or absence of Tregs might predispose to an uncontrolled adaptive immune reaction with severe lung damage and COPD.

Another population of T-cells with immunoregulatory properties are the CD8+ $\gamma \delta \mathrm{T}$-cells and are increased in smokers with normal lung function but not in smokers with COPD [22]. These findings point toward impaired immune regulation in smokers with COPD and to an effective control in smokers with normal lung function.

At this point, it is worth bringing attention to other types of cells with recently described immunoregulatory function, i.e. the M2 phenotype of the alveolar macrophages and the myeloid-derived suppressor cells (MDSCs), which may have potential roles in COPD and that could be targets for future investigation in smokers.

Alveolar macrophages in smokers seem to lose their proinflammatory characteristics, showing a decrease in phagocytic activity, production of inflammatory mediators and nitric oxide production, etc. [23]. This could reflect the recently described skewing of the classical M1 pro-inflammatory phenotype toward the alternative M2 phenotype with antiinflammatory, profibrotic repair properties in a majority of, but not all, smokers with COPD [24, 25]. This skewing toward the M2 phenotype could possibly be another factor tending to suppress the inflammatory process and even predispose to the development of malignancies in some smokers.

The MDSCs [26] are a set of cells described first in animal models and patients with advanced stages of cancer, and recently found even in patients with early cancer [27, 28], which by suppressing the protective immune response to malignant cells may promote the progression of the tumour and the development of metastasis [26]. However, there is strong evidence that these cells are also increased and play a regulatory role in the immune responses in bacterial and parasitic infections, acute and chronic inflammation, autoimmunity, traumatic stress, surgical sepsis, and transplantation [29-31]. With the idea that MDSCs could conceivably also be present in a chronic inflammatory condition like COPD, we investigated and found, in a small group of subjects, that smokers with COPD have higher numbers of circulating MDSCs than controls (unpublished observations). Although these results are of potential interest, they are preliminary and definitely deserve further investigation.

It would then be conceivable that the different mechanisms described to control the different steps of the inflammatory response in an attempt to modulate the progression of the disease in smokers might result in some degree of immunosuppression, which might have untoward consequences. 


\section{ARE SMOKERS IMMUNOSUPPRESSED?}

There are no available epidemiological studies investigating the immune state of smokers as compared with nonsmokers, possibly because the assessment of immune readiness or level of immunosuppression is not easy to investigate as no clear and easy tests are available. However, there are numerous studies in mice and humans showing a widespread suppression of many immune functions in smokers [32, 33]. These effects include suppression of the effector function of PMNs, natural killer cells and macrophages [34-37], which have a compromised ability to phagocytose bacteria and apoptotic cells and to sense pathogen-associated molecular patterns [38, 39]. Furthermore, alveolar macrophages show a skewing of the M1 effector function toward an M2-type immune response with an anti-inflammatory profile and possible tumour promotion [24]. A reduced proliferation of T-cells in response to T-cell mitogens and suppression of killing by cytotoxic Tcells have also been documented [40,41]. Of interest, human smokers have a significant reduction in their serum immunoglobulin levels, which might account for smoking being an important confounding cause for morbidity during an influenza epidemic [32]. In support of these observations, mice that were chronically exposed to cigarette smoke were more susceptible to influenza viruses [32]. Furthermore, the antibody response to various antigens can be reduced significantly as a consequence of chronic exposure to cigarette smoke [33].

It is not clear at this point if all these effects on the immune system are the direct consequence of the numerous compounds found in cigarette smoke (nicotine is a well-known immune suppressor) or of the attempts of the immune system to regulate itself in the presence of a sustained inflammatory challenge, as we described previously. Probably, both effects could account for the suppression of different components of the immune response that have been described in smokers, which ought to be variable, i.e. effective in smokers without disease or with mild disease but completely eluded in severe COPD, where a florid adaptive immune reaction drives the lung destruction.

\section{WHAT IS THE PRICE TO PAY FOR COPD EVASION?}

The association between smoking, COPD and lung cancer has been well demonstrated [42], and it has been shown that the incidence of cancer is higher in patients with airflow obstruction than in nonobstructed smokers [43]. Several factors triggered by chronic exposure to cigarettes would favour the development of cancer in smokers, such as the injury and necrosis of epithelial cells produced by the exposure to cigarette smoke, which are known to release factors like high-mobility group protein B1, promoting inflammation but also tissue repair [44, 45] and potentially favouring the development of malignancies. Interestingly, the induction of cancer in animals by dominant oncogenes requires the presence of tissue injury and subsequent regeneration [46].

An essential defense against cancer development is the activity of the immune system, encounters of which with a nascent tumour can bring about the elimination of the cancer. The increased prevalence of cancers in immunosuppressed transplant patients attests to the importance of the immune system in controlling the development of cancer $[47,48]$. Furthermore, the beneficial effect of enhanced immunity has also been shown in a population study in which subjects with a high or medium degree of natural cytotoxicity had a significantly lower risk of cancer than subjects whose lymphocytes had a low degree of cytotoxicity [49].

In favour of this contribution by the immune system to the development of lung cancer and COPD is a recent report by HeMminKi et al. [50]. Those authors examined the associations of autoimmune diseases with COPD and cancer, in order to determine the risk of COPD and cancer in persons who had been hospitalised for autoimmune diseases. Their findings showed that a great number of autoimmune diseases (18 out of 29 cases) were associated with COPD, but only a minority had an association with cancer. Furthermore, there was a remarkable lack of excess lung cancer in patients with autoimmune disease who developed COPD, suggesting that the defined autoimmunity might contribute to the development of COPD but also to the suppression of malignancy.

All things considered, it would not be surprising to find that smokers who evade the development of severe COPD by suppressing their immune system would have a higher incidence of lung cancer than smokers who develop severe COPD, in which a florid adaptive immune response and, probably, autoimmunity are present. Although high immunity would predispose to the development of severe COPD, it could also help prevent the development of cancer in these patients. The opposite could occur with the "evaders", who would develop less severe COPD, but might have to pay the price of a higher probability for the development of cancer in the lung and, possibly, other sites. Our recent work showing that smokers with severe COPD had a lower incidence of lung cancer than smokers with mild COPD supports this hypothesis [51].

In conclusion, it might be worthwhile to start thinking about why smokers evade COPD, rather than why they develop COPD. An inflammatory response to cigarette smoke in the lung seems to be the key for the development of COPD; thus, it is not surprising that those smokers who evade or have milder COPD have reduced inflammation. Inflammation is necessary, but could be harmful; thus, dampening inflammation on time is an essential protective mechanism in all types of inflammatory conditions, which also seems to be working in smokers who evade COPD. The failure of the blockade would result in chronic adaptive immune inflammation and, possibly, autoimmunity, as in the case of severe COPD. However, dampening of inflammation can render smokers relatively immunosuppressed, which may carry untoward consequences. Are dampening mechanisms failing in smokers who develop COPD? Why are they failing? A better understanding of this process could improve our knowledge of the immune-mediated inflammatory response in smokers and, thus, the mechanisms underlying the development of COPD.

\section{STATEMENT OF INTEREST}

A statement of interest for M. Saetta can be found at www.erj. ersjournals.com/site/misc/statements.xhtml

\section{REFERENCES}

1 Cosio MG, Saetta M, Agusti A. Immunologic aspects of chronic obstructive pulmonary disease. N Engl J Med 2009; 360: 2445-2454. 
2 Brent L. The 50th anniversary of the discovery of immunologic tolerance. N Engl J Med 2003; 349: 1381-1383.

3 Finkelstein R, Fraser RS, Ghezzo H, et al. Alveolar inflammation and its relation to emphysema in smokers. Am J Respir Crit Care Med 1995; 152: 1666-1672.

4 Saetta M, Di Stefano A, Turato G, et al. CD8+ T-lymphocytes in peripheral airways of smokers with chronic obstructive pulmonary disease. Am J Respir Crit Care Med 1998; 157: 822-826.

5 Boehmer H, Melchers F. Checkpoints in lymphocyte development and autoimmune disease. Nat Immunol 2010; 11: 14-20.

6 Mueller D. Mechanisms maintaining peripheral tolerance. Nat Immunol 2010; 11: 21-27.

7 Torres-Aguilar H, Blank M, Jara LJ, et al. Tolerogenic dendritic cells in autoimmune diseases: crucial players in induction and prevention of autoimmunity. Autoimmun Rev 2010; 10: 8-17.

8 Guerassimov A, Takubo Y, Turcotte A, et al. The development of emphysema in cigarette smoke exposed mice is strain dependent. Am J Respir Crit Care Med 2004; 170: 974-980.

9 Arend WP. The balance between IL-1 and IL-1Ra in disease. Cytokine Growth Factor Rev 13 2002; 323-340.

10 Han J, Ulevitch RJ. Limiting inflammatory responses during activation of innate immunity. Nat Immunol 2005; 6: 1182-1189.

11 Anderson P. Post-transcriptional regulons coordinate the initiation and resolution of inflammation. Nat Rev Immunol 2010; 10: 24-35.

12 Henson P. Dampening inflammation. Nat Immunol 2005; 6: $1179-1181$.

13 Serhan CN. Resolution phase of inflammation: novel endogenous anti-inflammatory and proresolving lipid mediators and pathways. Annu Rev Immunol 2007; 25: 101-137.

14 Tang $Q$, Bluestone JA. The Foxp3+ regulatory $\mathrm{T}$ cell: a jack of all trades, master of regulation. Nat Immunol 2008; 9: 239-244.

15 Jiang H, Chess L. An integrated view of suppressor T cell subsets in immunoregulation. J Clin Invest 2004; 114: 1198-1208.

16 Lane N, Robins RA, Corne $\mathrm{J}$, et al. Regulation in chronic obstructive lung disease: the role of regulatory T-cells and Th17 cells. Clin Sci 2010; 119: 75-86.

17 Smyth LJC, Starkey C, Vestbo J, et al. CD4-regulatory cells in COPD patients. Chest 2007; 132: 156-163.

18 Plumb J, Smyth LJC, Adams HR, et al. Increased T-regulatory cells within lymphocyte follicles in moderate COPD. Eur Respir J 2009; 34: 89-94.

19 Barcelo B, Pons J, Ferrer JM, et al. Phenotypic characterisation of T-lymphocytes in COPD: abnormal CD4+CD25+ regulatory Tlymphocyte response to tobacco smoking. Eur Respir J 2008; 31: 555-562.

20 Lee SH, Goswami S, Grudo A, et al. Antielastin autoimmunity in tobacco smoking-induced emphysema. Nat Med 2007; 13: 567-569.

21 Isajevs S, Taivans I, Strazda G, et al. Decreased FOXP3 expression in small airways of smokers with COPD. Eur Respir I 2009; 33: 61-67.

22 Pons J, Sauleda J, Ferrer JM, et al. Blunted $\gamma \delta \mathrm{T}-$ lymphocyte response in chronic obstructive pulmonary disease. Eur Respir J 2005; 25: 441-446.

23 Biswas SK, Mantovani A. Macrophage plasticity and interaction with lymphocyte subsets: cancer as a paradigm. Nat Immunol 2010; 11: 889-896.

24 Shaykhiev R, Krause A, Salit J, et al. Smoking-dependent reprogramming of alveolar macrophage polarization: implication for pathogenesis of COPD. J Immunol 2009; 183: 2867-2883.

25 Woodruff P. A distinctive alveolar macrophage activation state induced by cigarette smoking. Am J Respir Crit Care Med 2005; 172: 1383-1392.

26 Dolcetti L, Marigo I, Mantelli B, et al. Myeloid-derived suppressor cell role in tumor-related inflammation. Cancer Lett 2008; 267: 216-225.

27 Diaz-Montero $M$, Labib Salem $M$, Nishimura $M$, et al. Increased circulating myeloid-derived suppressor cells correlate with clinical cancer stage, metastatic tumor burden, and doxorubicin-cyclophosphamide chemotherapy. Cancer Immunol Immunother 2009; 58: 49-59.

28 Clark CE, Hingorani SR, Mick R, et al. Dynamics of the immune reaction to pancreatic cancer from inception to invasion. Cancer Res 2007; 67: 9518-9527.

29 Bunt SK, Clements VK, Hanson EM, et al. Inflammation enhances myeloid-derived suppressor cell crosstalk by signaling through Toll-like receptor 4. J Leukocyte Biol 2009; 85: 996-1004.

30 Gabrilovich DI, Nagaraj S. Myeloid-derived-suppressor cells as regulators of the immune system. Nat Rev Immunol 2009; 9 ; 162-174.

31 Ezernitchi AV, Vaknin I, Cohen-Daniel L, et al. TCR $\zeta$ downregulation under chronic inflammation is mediated by myeloid suppressor cells differentially distributed between various lymphatic organs. J Immunol 2006; 177: 4763-4772.

32 Sopori M. Effects of cigarette smoke on the immune system. Nature Rev Immunol 2002; 2: 372-377.

33 Stämpfli MR, Anderson GP. How cigarette smoke skews immune responses to promote infection, lung disease and cancer. Nature Rev Immunol 2009; 9: 377-384.

34 Tollerud DJ, Clark JW, Brown LM, et al. Association of cigarette smoking with decreased numbers of circulating natural killer cells. Am Rev Respir Dis 1989; 139: 194-198.

35 Mian MF, Lauzon NM, Stämpfli MR, et al. Impairment of human NK cell cytotoxic activity and cytokine release by cigarette smoke. J Leukoc Biol 2008; 83: 774-784.

36 Berenson CS, Garlipp MA, Grove LJ, et al. Impaired phagocytosis of nontypeable Haemophilus influenzae by human alveolar macrophages in chronic obstructive pulmonary disease. J Infect Dis 2006; 194: $1375-1384$

37 Hodge S, Hodge G, Ahern J, et al. Smoking alters alveolar macrophage recognition and phagocytic ability: implications in chronic obstructive pulmonary disease. Am J Respir Cell Mol Biol 2007; 37: 748-755.

38 Drannik AG, Pouladi MA, Robbins CS, et al. Impact of cigarette smoke on clearance and inflammation after Pseudomonas aeruginosa infection. Am J Respir Crit Care Med 2004; 170: 1164-1171.

39 Gaschler GJ, Zavitz CC, Bauer CM, et al. Cigarette smoke exposure attenuates cytokine production by mouse alveolar macrophages. Am J Respir Cell Mol Biol 2008; 38: 218-226.

40 Harrigan JA, Vezina CM, McGarrigle BP, et al. DNA adduct formation in precision?cut rat liver and lung slices exposed to benzo[a]pyrene. Toxicol Sci 2004; 77: 307-314.

41 Kalra R, Singh SP, Savage SM, et al. Effects of cigarette smoke on immune response: chronic exposure to cigarette smoke impairs antigen-mediated signaling in $\mathrm{T}$ cells and depletes $\mathrm{IP}_{3}$-sensitive $\mathrm{Ca}^{2+}$ stores. J Pharmacol Exp Ther 2000; 293: 166-171.

42 Mannino DM, Aguayo SM, Petty TL, et al. Low lung function and incident lung cancer in the United States: data from the First National Health and Nutrition Examination Survey follow-up. Arch Intern Med 2003; 163: 1475-1480.

43 Tockman MS, Anthonisen NR, Wright EC, et al. Airway obstruction and the risk for lung cancer. Ann Intern Med 1987; 106: 512-518.

44 Baraldo S, Saetta M, Cosio MG. For whom the "alarm" tolls: how we sense the danger of cigarette smoke and react to it. Am J Respir Crit Care Med 2010; 181: 879-880.

45 Ferhani N, Letuve S, Kozhich A, et al. Expression of high-mobility group box 1 and of receptor for advanced glycation end products in chronic obstructive pulmonary disease. Am J Respir Crit Care Med 2010; 181: 917-927.

46 Dvorak HF. Tumors: wounds that do not heal. Similarities between tumor stroma generation and wound healing. $N$ Engl J Med 1986; 315: 1650-1659.

47 Rama I, Grinyó JM. Malignancy after renal transplantation: the role of immunosuppression. Nat Rev Nephrol 2010; 6: 511-519. 
48 Gutierrez-Dalmau A, Campistol, JM. Immunosuppressive therapy and malignancy in organ transplant recipients: a systematic review. Drugs 2007; 67: 1167-1198.

49 Herbst RS, Heymach JV, Lippman SM. Molecular origins of cancer. Lung cancer. N Engl J Med 2008; 359: 1367-1380.
50 Hemminki K, Liu X, Ji J, et al. Subsequent COPD and lung cancer in patients with autoimmune disease. Eur Respir J 2011; 37: 463-464.

51 Torres JP, Marín JM, Casanova C, et al. Lung cancer in patients with COPD: incidence and predicting factors. Am J Respir Crit Care Med 2011; 184: 913-919. 